This item was submitted to Loughborough's Research Repository by the author.

Items in Figshare are protected by copyright, with all rights reserved, unless otherwise indicated.

\title{
In situ monitoring of laser-induced periodic surface structures formation on polymer films by grazing incidence small-angle X-ray scattering
}

PLEASE CITE THE PUBLISHED VERSION

http://dx.doi.org/10.1021/acs.langmuir.5b00285

\section{PUBLISHER}

(c) American Chemical Society

VERSION

AM (Accepted Manuscript)

\section{PUBLISHER STATEMENT}

This work is made available according to the conditions of the Creative Commons Attribution-NonCommercialNoDerivatives 4.0 International (CC BY-NC-ND 4.0) licence. Full details of this licence are available at: https://creativecommons.org/licenses/by-nc-nd/4.0/

\section{LICENCE}

CC BY-NC-ND 4.0

\section{REPOSITORY RECORD}

Rebollar, Esther, Daniel R. Rueda, Ignacio Martin-Fabiani, Alvaro Rodriguez-Rodriguez, Mari-Cruz GarciaGutierrez, Giuseppe Portale, Marta Castillejo, and Tiberio A. Ezquerra. 2017. "In Situ Monitoring of Laserinduced Periodic Surface Structures Formation on Polymer Films by Grazing Incidence Small-angle X-ray Scattering". figshare. https://hdl.handle.net/2134/26693. 


\section{In situ monitoring of Laser Induced Periodic Surface}

\section{Structures formation on polymer films by Grazing}

\section{Incidence Small Angle X-ray Scattering}

Esther Rebollar ${ }^{1 *}$, Daniel R. Rueda ${ }^{2}$, Ignacio Martín-Fabiani ${ }^{2,+}$ Álvaro Rodríguez-Rodríguez ${ }^{2}$, Mari-Cruz García-Gutiérrez ${ }^{2}$, Giuseppe Portale ${ }^{3}$, Marta Castillejo $^{1}$ and Tiberio A. Ezquerra ${ }^{2}$

${ }^{1}$ Instituto de Química Física Rocasolano (IQFR-CSIC), Serrano119, 28006 Madrid, Spain.

${ }^{2}$ Instituto de Estructura de la Materia (IEM-CSIC), Serrano 121, 28006 Madrid, Spain.

${ }^{3}$ DUBBLE@ESRF, Netherlands Organisation for Scientific Research (N.W.O.), CS40220, 38043, Grenoble, Cedex 9, France.

The formation of Laser Induced Periodic Surface Structures (LIPSS) on model spin-coated polymer films has been followed in situ by Grazing Incidence Small Angle X-ray Scattering (GISAXS) using synchrotron radiation. The samples were irradiated at different repetition rates ranging from 1 up to $10 \mathrm{~Hz}$ by using the 4th harmonic of a Nd:YAG laser $(266 \mathrm{~nm})$ with pulses of 8 ns. Simultaneously GISAXS patterns were acquired during laser irradiation. The variation of both the GISAXS signal with the number of pulses and the LIPSS period with laser irradiation time is revealing key kinetic aspects of the nanostructure formation process. By considering 
LIPSS as one-dimensional paracrystalline lattice and using a correlation found between the paracrystalline disorder parameter, $g$, and the number of reflections observed in the GISAXS patterns, the variation of the structural order of LIPSS can be assessed. The role of the laser repetition rate in the nanostructure formation has been clarified. For high pulse repetition rates (i.e. $10 \mathrm{~Hz}$ ), LIPSS evolve in time to reach the expected period matching the wavelength of the irradiating laser. For lower pulse repetition rates LIPSS formation is less effective and the period of the ripples never reaches the wavelength value. Results support and provide information on the existence of a feedback mechanism for LIPSS formation in polymer films.

\section{INTRODUCTION}

The surface properties of polymers can be of crucial importance in order to provide certain functionalities. In particular, the controlled micro- and nanostructuring of polymer surfaces is a useful approach aiming to create functional substrates. Different techniques such as those based on solution flow and evaporation, low energy ion beam erosion and advanced lithography have been proposed in order to produce superficial nanostructures on polymers. ${ }^{1-7} \mathrm{~A}$ current trend however is looking for alternative processes to lithography aiming to avoid the necessity of demanding experimental conditions, like clean rooms, high vacuum or complex mask fabrication among others. Formation of laser induced periodic surface structures (LIPSS) has been observed on the surface of different materials, such as metals, semiconductors and dielectrics upon irradiation with lasers at different wavelengths from ultraviolet to infrared and with different pulse durations from nanoseconds to femtoseconds. ${ }^{8-13}$ In the case of polymers, irradiation with a linearly polarized laser beam induces self-organized ripple structure formation within a narrow fluence range well below the ablation threshold. ${ }^{14-18}$ LIPPS can be prepared in both spin-coated and free standing polymer films. ${ }^{19}$ This fact makes LIPSS a potential method to obtain large 
surface area and good quality samples. The advantage of using laser based methods can be twofold. In the first place, the increased flexibility, since the periodicity of the structures can be tuned by the laser wavelength. In the second place, lasers treatment can easily be extended to large surface. An example of these structures is shown in Figure 1 for a thin film of poly(trimethylene terephthalate) (PTT) where LIPSS are parallel to the laser polarization vector.

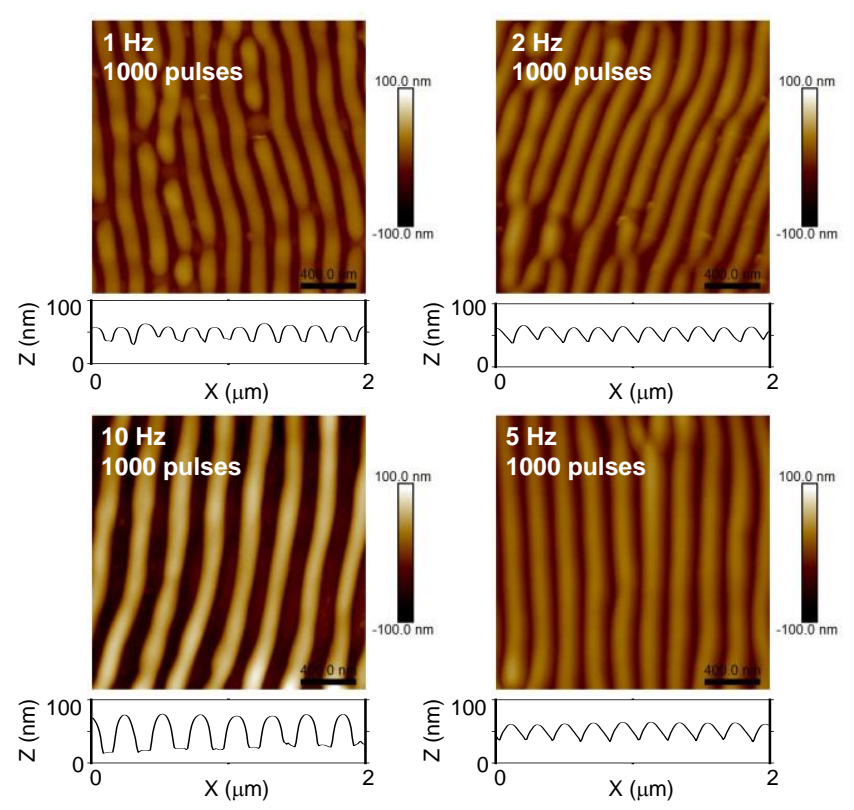

Figure 1. AFM height images $\left(2 \times 2 \mu \mathrm{m}^{2}\right)$ clockwise represented as a function of repetition rate for irradiation with a linearly polarized beam at $266 \mathrm{~nm}$ using 1000 pulses and $7 \mathrm{~mJ} / \mathrm{cm}^{2}$. The height profile along a $2 \mu \mathrm{m}$ line perpendicular to the ripples is shown below every image.

The period of the ripples $L$ produced by a laser beam of wavelength $\lambda$, reaching the surface of a material of refraction index $n$, at an incidence angle $\theta$ respect to the surface normal, can be described by the relation $L=\lambda /(n-\sin \theta)$. LIPSS are formed on the material surface as a result of the interference between the incoming and the surface-scattered waves, in such a way that an inhomogeneous intensity distribution, together with a feedback mechanism, results in the 
enhancement of the modulation depth. ${ }^{20,21}$ Recently, it has been proposed ${ }^{15}$ that for obtaining LIPSS it is important that the polymer absorbs efficiently at the laser wavelength used for irradiation. LIPSS formation in polymer substrates upon irradiation with nanosecond pulses is mediated by heating of the polymer surface. The formation of LIPSS in general requires that the polymer segments reach enough mobility to be able to rearrange. This can happen above the glass transition, if the polymer is amorphous or above the melting point if the polymer is semicrystalline. Heating provides enough polymer chain mobility to rearrange the material in the surface giving rise to the superficial height modulation. ${ }^{15,22,23}$ However, the whole mechanism responsible for LIPSS formation is still not completely understood in polymers.

Structural characterization of nanopatterned surfaces is commonly undertaken by microscopy techniques like scanning electron microscopy (SEM) and atomic force microscopy (AFM). ${ }^{24,25}$ The visualization of nanostructures in real space is advantageous since it allows direct assessment of pattern morphology. However, quite frequently microscopies allow visualization restricted to small and superficial areas of the sample. Moreover, these techniques become unpractical upon dealing with evaluation of fast processes in real time. For these cases, the use of X-ray scattering techniques with synchrotron radiation can be very useful as they allow obtaining reciprocal space structural information in the millisecond time range. ${ }^{26}$ In order to perform X-ray scattering on surface nanostructures it is very convenient to work under total X-ray reflection conditions. ${ }^{27-30}$ The X-ray pattern provides statistical information integrated over a large sample area covered by the footprint of the incident beam on the material surface. Therefore, the scattering pattern can deliver structural information averaged over an area of several hundreds of microns. Previous studies on the evolution with time/number of laser pulses of LIPSS have been carried out by irradiating a given sample with a certain number of pulses and by performing the 
structural characterization ex-situ. ${ }^{15,18,22}$ This procedure limits the amount of samples and rules out in situ analysis and the investigation of laser irradiation at high repetition rate.

In this work we have accomplished in situ characterization of LIPSS formation on the surface of a model polymer film by using Grazing Incidence Small Angle X-ray Scattering (GISAXS) with synchrotron radiation. By this novel approach the characterization of the nanostructure formation process has been possible for a laser pulse repetition rate up to $10 \mathrm{~Hz}$. Additionally, the role of pulse repetition rate on LIPSS formation has been studied, since the time elapsed between consecutive pulses may have an influence on the effective nanostructure formation process.

\section{EXPERIMENTAL}

\subsection{Materials and samples preparation}

The model polymer used was poly(trimethylene terephthalate) (PTT) which is a linear aromatic polyester based on the terephthalic acid, as some others like poly(ethylene terephthalate) (PET) and poly(butylene terephthalate) (PBT). PTT was synthesized by polycondensation as previously described, ${ }^{31}$ yielding a number molecular weight of $M_{n}=31294$ $\mathrm{g} / \mathrm{mol}$ and a polydispersity of $M_{w} / M_{n}=2.22$, as determined by size exclusion chromatography. PTT is a semicrystalline polymer, with a melting temperature of $T_{m}=229{ }^{\circ} \mathrm{C}$ and a glass transition temperature, determined by calorimetry, ${ }^{32}$ of $T_{g}=44{ }^{\circ} \mathrm{C}$. Polymer thin films were prepared by spin-coating on silicon wafers (100) (Wafer World Inc.) polished on one side. The wafers were previously cleaned with acetone and isopropanol. PTT was solved in trifluoroacetic acid (Sigma-Aldrich, reagent grade $\geq 98 \%$ ) with a concentration of $20 \mathrm{~g} / \mathrm{L}$. A fixed amount of $0.1 \mathrm{~mL}$ of polymer solution was instantly dropped by a syringe on a square (typically $2 \times 2 \mathrm{~cm}^{2}$ ) silicon substrate placed in the center of a rotating metallic horizontal plate. A rotation speed of 
$2380 \mathrm{rpm}$ was kept during $30 \mathrm{~s}$. Spin-coated polymer films with a thickness of about $157 \pm 24 \mathrm{~nm}$ and extremely flat surface (mean surface roughness $R_{a} \leq 1 \mathrm{~nm}$ ), as measured by AFM, are typically obtained under these conditions ${ }^{22}$.

\subsection{Simultaneous GISAXS-LIPSS experimental set-up}

For LIPSS formation on the polymer spin-coated films we used the linearly polarized laser beam of a Q-switched Nd:YAG laser (Lotis TII LS-2131M) with pulses of $8 \mathrm{~ns}$ at repetition rates in the 1-10 Hz range. The fourth harmonic at $266 \mathrm{~nm}$ was used as this laser wavelength falls into the absorption band of PTT, being its linear absorption coefficient ${ }^{22} \alpha_{266}=26 \cdot 10^{3} \mathrm{~cm}^{-1}$. The monitoring of LIPSS formation in situ was carried out by using synchrotron radiation at the DUBBLE beamline $e^{33}$ of the European Synchrotron Radiation Facility (ESRF, Grenoble, France). GISAXS patterns were acquired while simultaneous laser irradiation on the sample was taking place. A scheme of the set-up is shown in Figure 2.

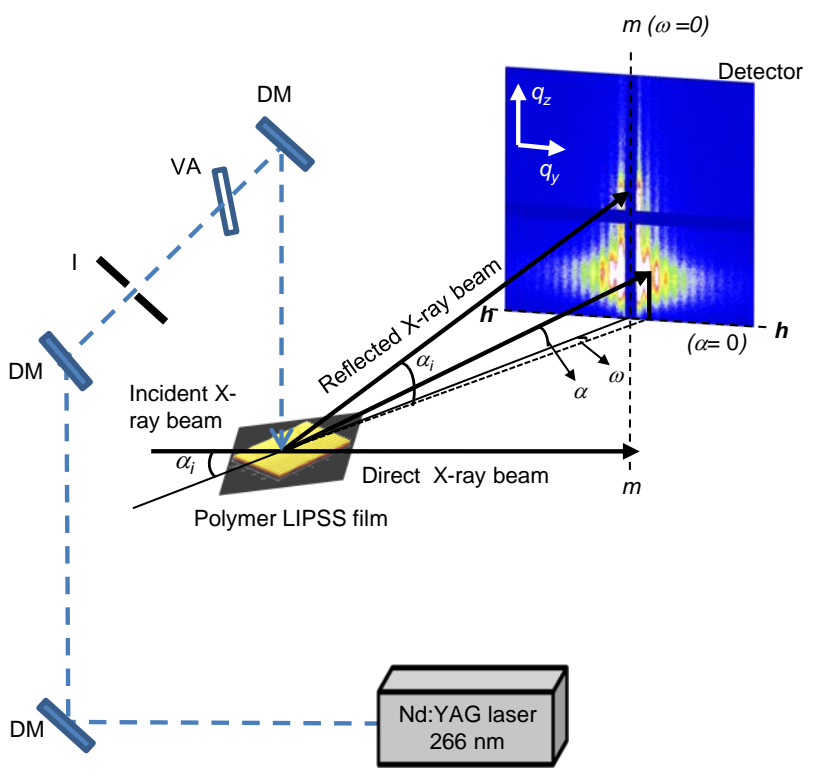

Figure 2. Scheme of the experimental set-up for in situ monitoring of LIPSS formation by GISAXS. The laser beam (blue dashed line) is directed by a collection of three dichroic mirrors (DM) to the sample surface so that it impinges vertically on the sample at normal incidence. The 
laser beam size is controlled by an iris (I) and its intensity by a variable attenuator (VA). The Xray beam (black line) reaches the sample which is positioned in grazing incidence conditions ${ }^{22,}$ ${ }^{34}$. The scattering plane, containing both the direct and the specular beams intersects the detector plane along the meridian, $m-m$ line, of the GISAXS pattern. The horizon, $h-h$ line, is the intersection between the sample plane and the plane of the detector.

The sample is mounted horizontally and parallel to the X-ray beam in a grazing incidence geometry ${ }^{22,34}$. A precise alignment of the laser beam is required in order to reach the sample surface perpendicularly. Furthermore, the laser polarization vector, which determines the axis of the ripples, must be parallel to the X-ray beam direction, since LIPSS are formed parallel to the laser polarization direction. The use in our case of three dichroic mirrors for control of the laser beam direction facilitates this condition. A laser fluence of $7 \mathrm{~mJ} / \mathrm{cm}^{2}$ was used for all the experiments which were carried out at four different repetition rates of 1, 2, 5, and $10 \mathrm{~Hz}$. For the sake of comparison, all the samples were irradiated with 2000 pulses. A laser irradiated area of 5 $\mathrm{mm}$ diameter was selected with an iris placed in front of the sample. For the GISAXS measurements an X-ray wavelength $\lambda=0.124 \mathrm{~nm}$ and a beam size $(\mathrm{HxV})$ of $1.1 \mathrm{x} 0.5 \mathrm{~mm}^{2}$ were used. Spatial overlap between the laser and X-ray beams was achieved in such a way that the central part of the laser irradiated spot was completely covered by the footprint of the X-ray beam. The GISAXS patterns were recorded by a Pilatus detector (981x1043 pixels, $172.0 \mu \mathrm{m}$ pixel size) which was located at $3.949 \mathrm{~m}$ from the sample position. The sample was aligned with its surface horizontal and parallel to the X-ray beam and at a height which intercepted half of the beam intensity. Then, for the GISAXS measurements, the sample was rotated in order to reach an incidence angle between the sample surface and the beam of $\alpha_{i}=0.4^{\circ}$. GISAXS patterns show 
the scattering intensity collected by the detector above the horizon line (exit angle $\alpha=0^{\circ}$ ) which corresponds to the intersection of the sample plane with the detector plane. A vertical plane (known as the scattering plane) is defined by both the incident and reflected beams which intersects the detector along the meridian, $m-m$ line $\left(\omega=0^{\circ}\right)$. Thus, each point $(y, z)$ of the pattern is defined by the angular coordinates $(\omega, \alpha)$ or by means of the corresponding components $\left(q_{y}\right.$, $q_{z}$ ) of the reciprocal scattering vector $q$. The angular and reciprocal coordinates are related by the expressions $^{35} q_{y}=(2 \pi / \lambda) \sin \omega \cos \alpha$ and $q_{z}=(2 \pi / \lambda)\left(\sin \alpha_{i}+\sin \alpha\right)$, where $\lambda$ is the X-ray wavelength.

In order to follow LIPSS formation in situ, both laser irradiation and GISAXS acquisition were simultaneously activated. Acquisition time for the GISAXS patterns for laser irradiation at 1, 2 and $5 \mathrm{~Hz}$ was $5 \mathrm{~s}$ while $3 \mathrm{~s}$ was selected for the first seven patterns for the faster repetition rate of $10 \mathrm{~Hz}$. GISAXS patterns were analyzed by the Fit2D software. ${ }^{36}$

\section{RESULTS}

A representative example of the in situ monitoring of LIPSS formation upon laser irradiation is shown in Figure 3 for a laser repetition rate of $5 \mathrm{~Hz}$. This figure shows a sequence of GISAXS patterns during irradiation of a PTT film as a function of both the number of pulses and the corresponding real experimental time. The first pattern taken after laser irradiation with 25 pulses $(5 \mathrm{~s})$ is still the characteristic one for a spin-coated film in absence of relevant structure in the angular range investigated ${ }^{37}$. After irradiation with 200 pulses (40 s), the GISAXS pattern starts exhibiting clear vertical diffraction maxima. For higher number of pulses the vertical diffraction maxima increase both in number and in intensity. 


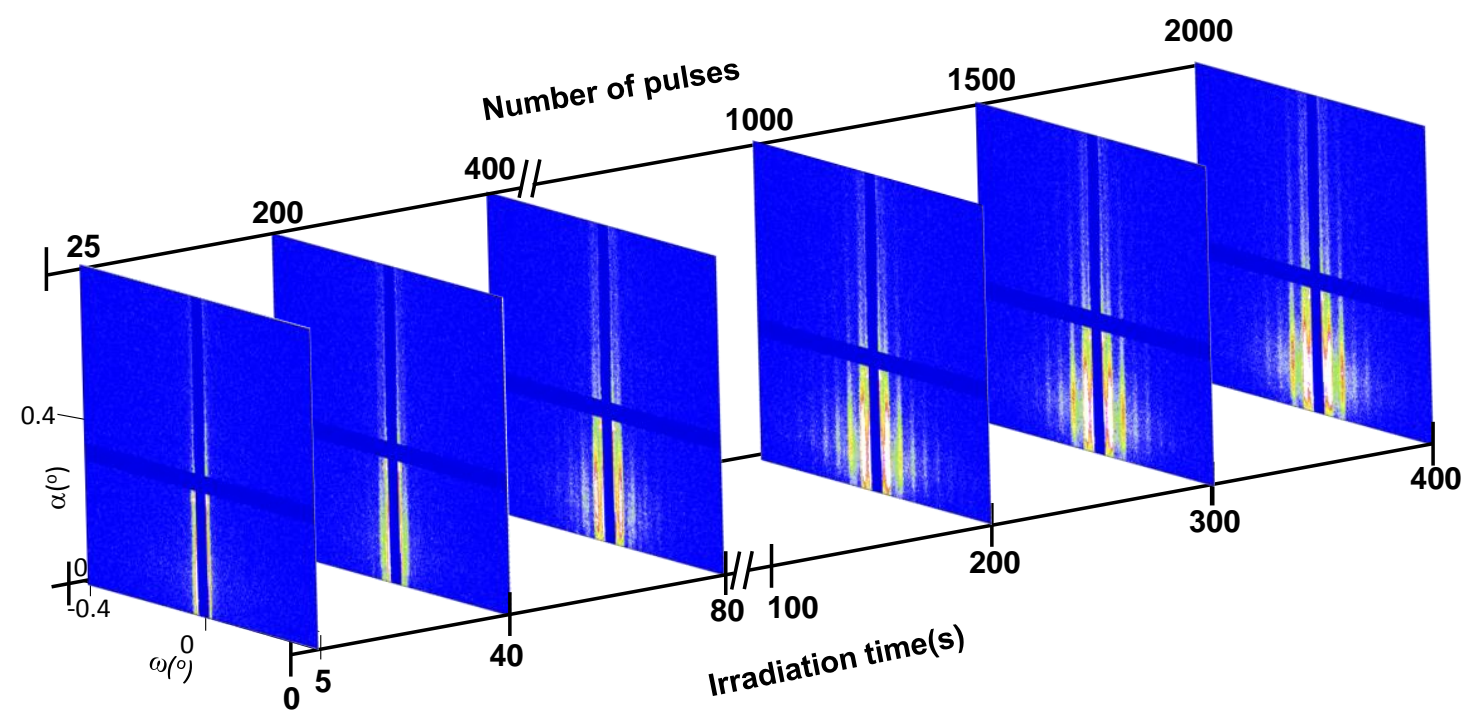

Figure 3. Sequence of GISAXS patterns acquired in situ as a function of both time and number of pulses for a PTT film laser irradiated with a laser repetition rate of $5 \mathrm{~Hz}$.

The influence of the laser repetition rate on LIPSS formation is illustrated in Figure 4 where selected GISAXS patterns corresponding to irradiation with 1000 laser pulses are shown. A first look of the patterns of Figure 4 reveals that the separation of the vertical diffraction maxima is similar for the three lower repetition rates used $(1,2$ and $5 \mathrm{~Hz})$ and larger than that observed for $10 \mathrm{~Hz}$. The morphology as revealed by AFM of the films with LIPSS corresponding to these patterns, is shown in Figure 1. 


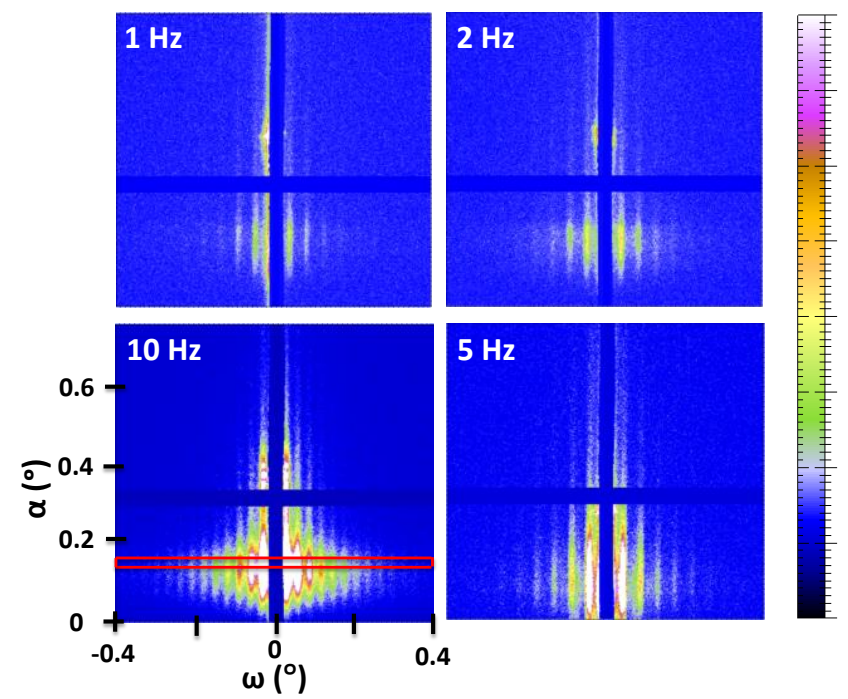

Figure 4. GISAXS patterns clockwise represented as a function of increasing laser repetition rate for the same number of 1000 laser pulses. The red horizontal box on the pattern at $10 \mathrm{~Hz}$ indicates the region where vertically integrated intensity profiles were taken.

From the GISAXS patterns we have extracted horizontal intensity profiles along $\omega$ for constant $\alpha$ values. These were obtained by vertical integration of a horizontal strip (11 pixel high) as the one shown in Figure 4 around the maximum of intensity of the vertical diffraction maxima $(\alpha \approx$ $0.15^{\circ}$ ). This angular region corresponds to the Yoneda peak, which is related to the critical angle of polymers for the X-rays ${ }^{38}$. The intensity profiles for a repetition rate of $2 \mathrm{~Hz}$ are shown in Figure 5a as a function of $\omega$. These data are qualitatively similar to those obtained for 1 and 5 Hz. Figure $5 \mathrm{~b}$ shows the corresponding intensity profiles for the sample irradiated at $10 \mathrm{~Hz}$. 

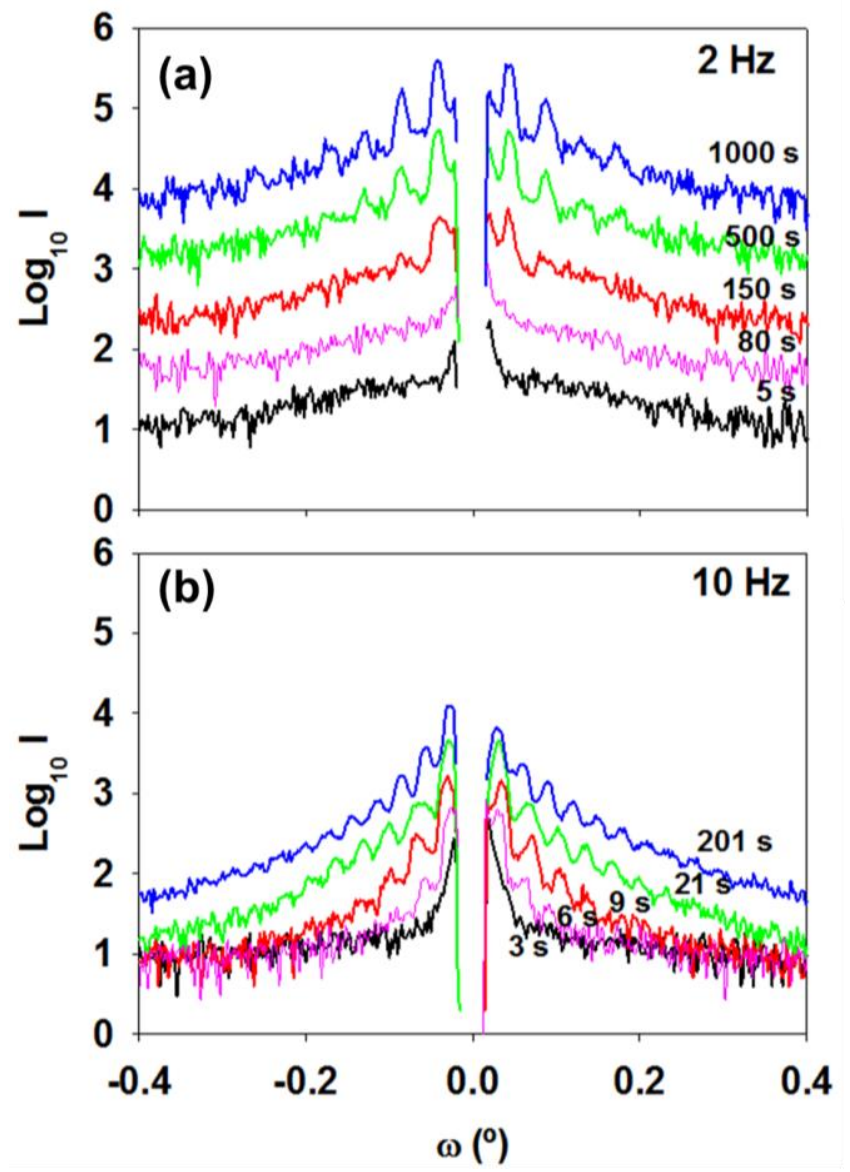

Figure 5. Selected GISAXS intensity profiles in logarithmic scale for laser irradiation at repetition rates of (a) $2 \mathrm{~Hz}$ and (b) $10 \mathrm{~Hz}$. The labels indicate the experimental real time at which the patterns were acquired. Curves for $2 \mathrm{~Hz}$ are shifted vertically by a half decade for clarity.

The period of the LIPSS can be derived from the lateral position of the first maxima, that is the nearest one to $\omega^{\circ}=0^{\circ}\left(q_{y}=0 \mathrm{~nm}^{-1}\right)$, using the expression: $L=2 \pi / q_{y}{ }^{\max }$. The outer maxima are consecutive orders of the first one. ${ }^{22}$ By comparing the intensity profiles for the samples irradiated at 2 and at $10 \mathrm{~Hz}$, it is clear that a larger period is obtained for the sample irradiated at $10 \mathrm{~Hz}$. This is corroborated by the analysis of the AFM images shown in Figure 1. Figure 6a 
presents the variation of the period extracted from the GISAXS analysis with the laser irradiation time for the samples irradiated at different repetition rates.

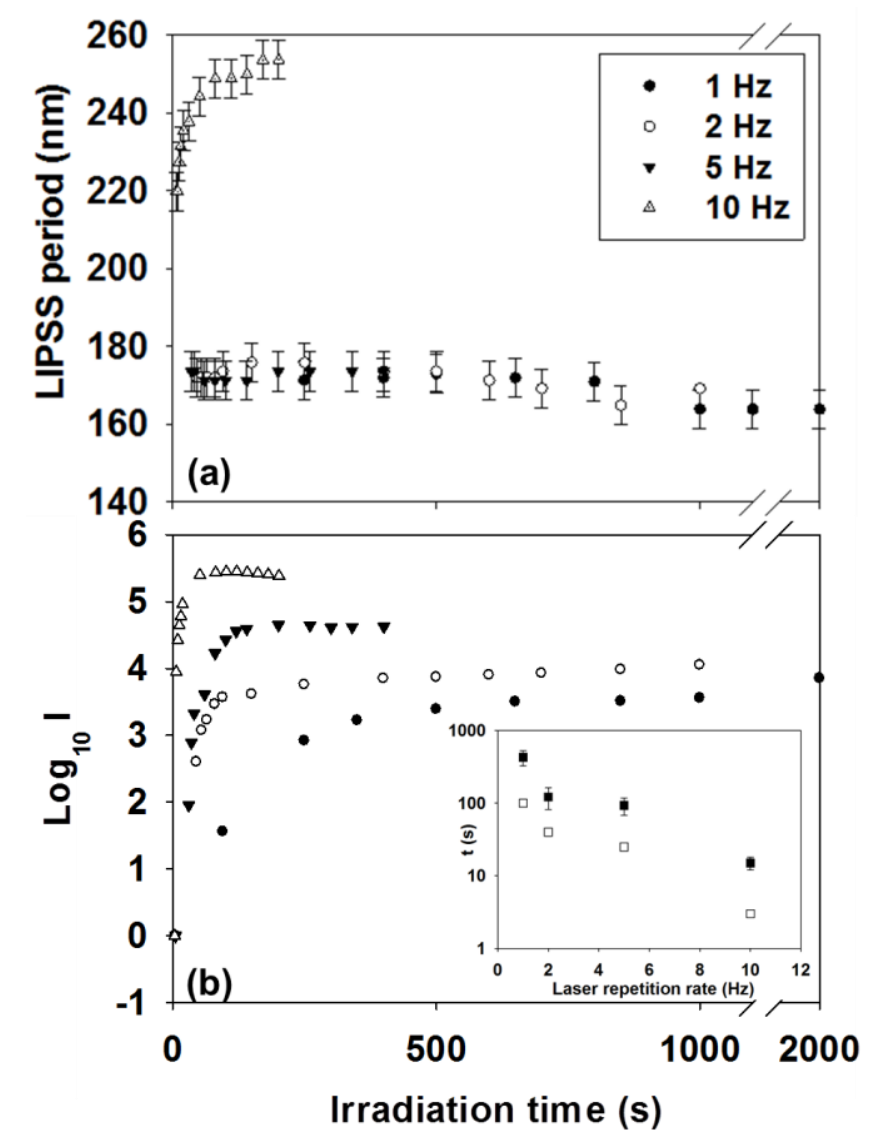

Figure 6. (a) Time dependence of the period of LIPSS developed on PTT films upon laser irradiation at different laser repetition rates as indicated. (b) Time dependence of the GISAXS intensity profiles (for the $\omega$ interval from $-0.4^{\circ}$ to $+0.4^{\circ}$ ), in logarithmic scale, at different repetition rates. The inset shows the half time ( $\mathbf{})$ and induction time ( $\square$ ) for LIPSS formation.

For the sample irradiated at $10 \mathrm{~Hz}$ the period increases with the number of pulses from a value around $220 \mathrm{~nm}$ and reaches a plateau with a value close to the laser wavelength $(266 \mathrm{~nm})$. For irradiation at lower repetition rates 1,2 and $5 \mathrm{~Hz}$, the period remains practically constant independently of the number of pulses. In this range, the period is significantly smaller than the 
wavelength of the laser. This observation is in good agreement with the period derived from analysis of the AFM images shown in Figure1 which yields values of $188 \pm 10,188 \pm 8,201 \pm 11$ and $259 \pm 8 \mathrm{~nm}$ for $1,2,5$ and $10 \mathrm{~Hz}$ respectively. In all the cases, the period obtained from GISAXS measurements is smaller than that derived from AFM analysis, as previously observed $^{15,22}$ although a good agreement within $\sim 20 \%$ is obtained between both period values. It is known that for nonhomogeneous systems the comparison between AFM Fourier transformed images, which produce essentially a two-dimensional power spectral density function, and diffraction patterns can exhibit slight mismatches ${ }^{39}$ since GISAXS averages over a sample volume usually larger than that visualized by AFM.

In order to compare the evolution with time of LIPSS formation for the different repetition rates, in Figure $6 \mathrm{~b}$ we have represented the total integrated intensity of the scattering profiles, like those shown in Figure 5, as a function of irradiation time. For the sake of comparison we have subtracted the integrated intensity of the intensity profile corresponding to the first GISAXS pattern for each experiment. Figure $6 \mathrm{~b}$ shows that for all the investigated repetition rates there is an initial time regime in which the logarithm of the integrated intensity rapidly increases followed by a second regime where it tends to stabilize. The initial regime is faster for a higher repetition rate. From these data it is possible to estimate the half time for LIPSS development, defined as the time at which half of the maximum intensity is reached. The inset in Figure $6 \mathrm{~b}$ shows that the half time for LIPSS formation decreases logarithmically with the repetition rate. Moreover, from the analysis of the GISAXS patterns we can infer an induction time for LIPSS formation, defined as the first time at which the first scattering vertical maxima start to be detected. These data have been also included in the inset of Figure $6 \mathrm{~b}$. As one sees, induction times exhibit similar repetition rate dependence as that of the development half times. 


\section{DISCUSSION}

\subsection{Order development in LIPSS}

As mentioned above, the number of vertical maxima present in the GISAXS patterns increases with the time of laser irradiation (Figure 3). In addition, the number of vertical maxima observed in a GISAXS pattern depends on the laser repetition rate for a similar number of pulses applied (Figure 4). The correlation between the number of reflections/peaks related to a given periodicity and the perfection of the lattice along such a periodicity is well known in crystallography ${ }^{40}$. In previous studies we showed that the GISAXS patterns obtained for LIPSS fabricated in polymer films can be understood by considering that the ripples constitute a one-dimensional paracrystalline lattice, ${ }^{15,22,41}$ where the long range order disappears gradually in a probabilistic way. $^{27,}{ }^{42-44}$ The probability of finding a particle at a periodic distance $L$ is defined by the Gaussian function $p(x)$ :

$$
p(x)=\frac{1}{\sigma \sqrt{2 \pi}} \exp \left[-\frac{(x-L)^{2}}{2 \sigma^{2}}\right]
$$

As the paracrystalline disorder parameter ${ }^{43} g=\sigma / L$ increases, the structural disorder of the lattice increases, while for very small $g$ values a $1 \mathrm{D}$ crystalline lattice is obtained. By using modelling tools it is possible to derive the $g$ value. Here, we have used the IsGISAXS ${ }^{45}$ software to generate the intensity profiles corresponding to theoretical one-dimensional lattices of known period, $L$, constituted by a series of elongated boxes as the scatterer motives. This model has been previously applied to LIPSS in films of different polymers including PTT. ${ }^{15,22,41}$ In this way we can generate different GISAXS patterns, that can be compared to those obtained experimentally, by changing the value of the paracrystallinity parameter $g$. As an example, Figure 7 shows a series of horizontal intensity profiles of different GISAXS patterns obtained by varying the $g$ value for a one dimensional paracrystalline lattice with a period $L=250 \mathrm{~nm}$ 
containing the same motive, a box of dimensions $2 R=100 \mathrm{~nm} ; H / R=0.3 ; W / R=10$, where $2 R$, $H$, and $W$ denote the width, the height, and the length of the box respectively.

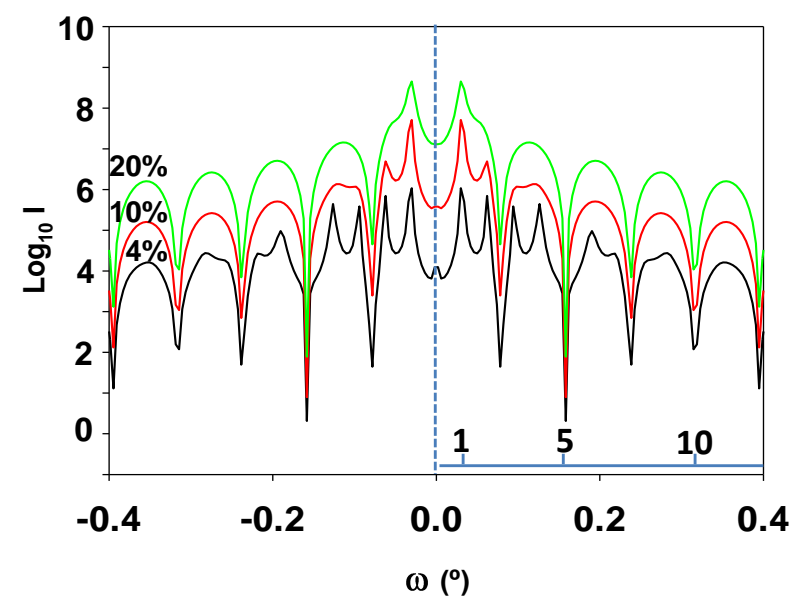

Figure 7. Intensity profiles from simulated GISAXS patterns with different paracrystalline parameter $g$ values: $4 \%$ (black), $10 \%$ (red), and $20 \%$ (green). The lattice period $L=250 \mathrm{~nm}$, and the box motive has the dimensions: $2 R=100 \mathrm{~nm}, H / R=0.3$ and $W / R=10$ where $2 R, H$, and $W$ denote the width, the height, and the length of the box respectively. The position of the first maximum associated to the structure factor and that of some consecutive orders are also labelled inside the graphic. Intensity profiles are vertically shifted by a decade for the sake of clarity.

In the intensity profiles one can distinguish two different types of maxima. The sharper ones are associated to the lattice structure factor while the rounded ones are attributed to the form factor of the scatterer. ${ }^{41}$ The maxima associated to the structure factor can be easily distinguished. It is observed that the number of peaks related to the structure factor decreases when the $g$ parameter increases. On the other hand, the position of the first minimum of intensity is related to the box width $(100 \mathrm{~nm})$. For this particular lattice the first minimum appears at 2.5 times the position of the first peak since $L / 2 R=2.5$. Then the $5^{\text {th }}$ order peak should be located on 
the second minimum of intensity. Thus, for $g=4 \%$ the number of structure peaks can be estimated as being between 8 or 9 (black curve in Figure 7). For $g=10 \%$ the number of peaks is between 3 and 4 (red curve) while for $g=20 \%$ only the first peak would be observed (green curve). Figure 8 shows the correlation between the paracrystalline disorder parameter $g$ and the number of peaks related to the structure factor for a series of lattices with the same box dimensions and different periods. For simulation, X-ray beam was considered parallel to the main box axis. It is observed that variation of the height of the box, $H$, does not significantly modify the shown trend.

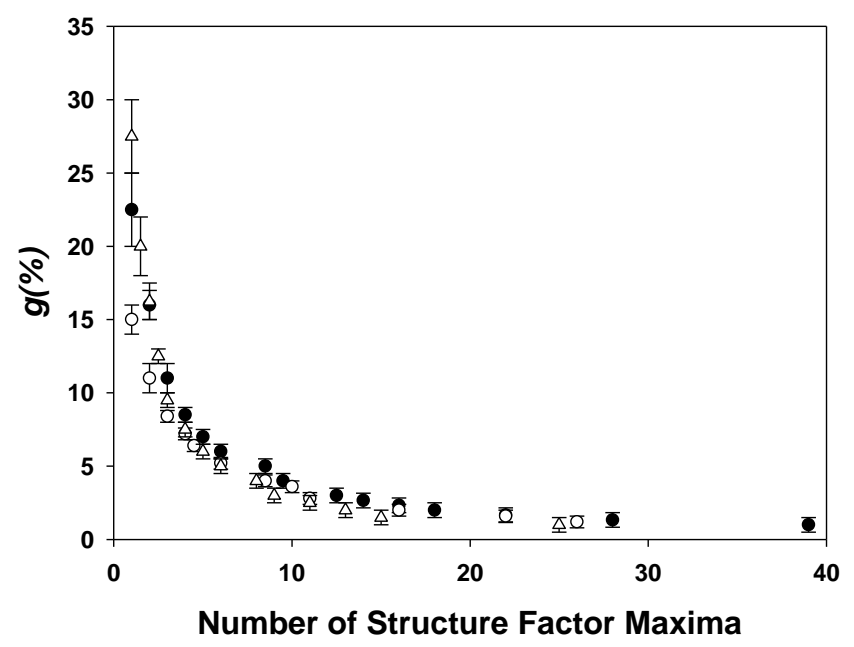

Figure 8. Correlation between the paracrystalline disorder parameter $g$ and the number of structure factor maxima corresponding to the horizontal intensity profiles derived from simulated GISAXS patterns for 1D-paracrystalline lattices of different period, $300 \mathrm{~nm}(\bullet), 250 \mathrm{~nm}(\circ)$ and $200 \mathrm{~nm}(\Delta)$. The scatterer motive is in all cases an elongated box of $100 \mathrm{~nm}$ width $(2 R)$ with $H / R$ $=0.3$ and $W / R=10$ being $H$ and $W$ the height and length of the box, respectively.

The results shown in Figure 8 indicate a hyperbolic correlation between the paracrystalline disorder parameter $g$ and the number of maxima associated to the structure factor. Thus, in a first 
approach it looks reasonable to use the correlation found in Figure 8 to experimentally derive the $g$ parameter from the number of vertical diffraction maxima observed in each GISAXS pattern acquired in the present experiments. The variation of the paracrystalline disorder parameter with the laser irradiation time is presented in Figure 9 for the different repetition rates used.

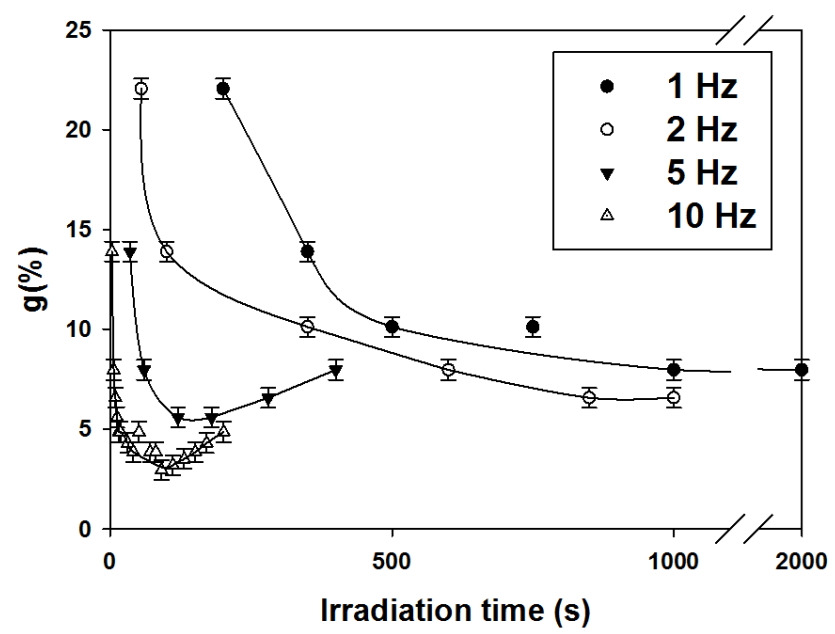

Figure 9. Paracrystalline disorder parameter, $g$, estimated by means of the correlation found in plot of Figure 8, from the number of vertical diffraction maxima observed in the GISAXS patterns as a function of the laser irradiation time for different repetition rates. The continuous lines are a guide for the eye.

According to the results of Figure 9, an initial decrease of $g$ is observed for all the repetition rates investigated. This reveals a progressive order improvement of LIPSS as the number of pulses applied to the polymer film increases. The improvement is clearly dependent on the laser repetition rate, being $10 \mathrm{~Hz}$ the one which renders the more perfect structure in terms of paracrystalline order. For 10 and $5 \mathrm{~Hz}$ the curves show a minimum. This would indicate that after the minimum further laser irradiation induces the deterioration of the ripples. For 1 and 2 $\mathrm{Hz}$ the $g$ parameter seems to reach a plateau after an irradiation time corresponding to about 
1000 pulses. Taking into account the results presented above we can say that the induced structural order on the surface of spin-coated polymer film by laser irradiation is strongly dependent on the laser repetition rate applied. The scattered intensity of the GISAXS patterns is related to the evolution of the nanostructure formation on the polymer film. The main structural feature is the formation of ripples as illustrated in Figure 1. In this figure it is observed that the height profiles clearly depend on the laser repetition rate. That means a very different corrugation of the samples which is maximum for $10 \mathrm{~Hz}$. The corrugation on the polymer surface is equivalent to an electronic density contrast which is responsible for the scattered intensity observed on the GISAXS patterns. ${ }^{46}$ On the one hand, when the electronic contrast is periodic like in LIPSS morphology several periodic scattering maxima are observed. On the other hand, the non-periodic electronic contrast would contribute to a continuous scattering. The latter contribution is particularly evident in the plots depicted in Figure 5b

\subsection{Mechanism of LIPSS formation}

Our results of in situ monitoring of LIPSS formation show that the dynamics of nanostructure formation is dependent on the laser repetition rate in the range $1-10 \mathrm{~Hz}$, as reflected by the trend observed for the half time and the induction time (inset of Figure 6b). In addition, the final values of the LIPSS periods are observed to be a function of the laser repetition rate (Figure 6a). These differences must be related to the mechanisms underlying LIPSS formation. It is commonly accepted that interference between the incident laser beam and the surface-scattered wave plays an important role in LIPSS formation. ${ }^{20,21}$ In this case, the irradiated surface scatters the incident beam which interferes with the surface wave resulting in a modulated distribution of the energy on the surface. ${ }^{47}$ Additionally, a feedback mechanism has been proposed to be involved, and LIPSS become fully developed only after a sufficient number of laser pulses have 
been applied to the polymer surface. ${ }^{15,48-50}$ However no information is available on the kinetics of LIPSS formation and the influence of pulse repetition rate has not been previously assessed in detail.

In principle one can consider the surface of a spin-coated polymer thin film at room temperature as having a profile randomly decorated with height variations caused by quenched, frozen-in, thermally excited capillary waves. ${ }^{51,52}$ These random height variations can be envisioned as the superposition of many different Fourier components with different spatial periods. ${ }^{47,53}$ In this case the roughness, $\sigma$, is given by: ${ }^{53}$ $\sigma=\sqrt{\sigma_{0}^{2}+\frac{k T}{2 \pi \gamma} \ln \left(\frac{l_{l}}{l_{s}}\right)} \quad$ (eq.2) where $\sigma_{o}$ is the molecular roughness, $\gamma$ is the surface tension, and $l_{s}$ and $l_{l}$ are, respectively, the shortest and longest wavelength of capillary waves that can be sustained at the liquid-vapor interface. The shortest wavelength is of the order of the distance between the atoms or molecules in the liquid and the longest is limited by the attractive interaction with the underlying substrate $^{53}$. Due to the fact that in our case the PTT film at room temperature is below the glass transition temperature $T_{g}\left(44^{\circ} \mathrm{C}\right)$, the film roughness is then dictated by the complex mechanism of solidification of the liquid solution during spin-coating. ${ }^{54}$

For polymer films in general, and for PTT in particular, it has been proposed that laser irradiation causes heating of the upper layer of the polymeric film. ${ }^{15,21,22}$ The corresponding temperature increase can be estimated by solving the one-dimensional heat conduction equation, as previously reported. ${ }^{15,22}$ For PTT, upon irradiation with a single nanosecond pulse at irradiation fluences above $4 \mathrm{~mJ} / \mathrm{cm}^{2}$, the temperature increases above $T_{g}$ reaching values of around 87 and $203{ }^{\circ} \mathrm{C}$ for fluences of 5 and $14 \mathrm{~mJ} / \mathrm{cm}^{2}$ respectively. ${ }^{22}$ Laser heating induces the transformation of the polymer from a glassy to a viscoelastic liquid state and therefore allows 
polymer segmental and chain dynamics. According to eq. 2 , the increase of temperature leads to an increase of surface roughness caused by capillary waves ${ }^{53}$. After laser induced local heating the polymer material cools down to its initial surface temperature. Surface inhomogeneities are expected to be enhanced as the next pulse would find a rougher surface thus facilitating the feedback mechanism. The time interval between successive pulses is $1,0.5,0.2$ and $0.1 \mathrm{~s}$ for repetition rates of $1,2,5$ and $10 \mathrm{~Hz}$ respectively. In our case, in situ monitoring of LIPSS formation by GISAXS serves to study the influence of time separation between successive laser pulses on both the period of the nanostructures and on the irradiation time (i.e., number of pulses) needed for LIPSS formation. The results show that for the repetition rates of 1,2 and 5 $\mathrm{Hz}$, the LIPSS period shows no significant changes with irradiation time (Figure 6a) for the number of pulses used in these experiments (up to 2000). However, for the highest repetition rate investigated, $10 \mathrm{~Hz}$, a clear evolution of the LIPSS period is observed as it increases with irradiation time until a plateau is reached for values close to the laser wavelength $(266 \mathrm{~nm})$. Additionally, for $10 \mathrm{~Hz}$ a much lower number of pulses (ca. 30) is needed for the appearance of LIPSS, in comparison to the number of pulses required (close or above 100) at lower repetition rates. The fact that the period increases for higher repetition rates, while the irradiation time and number of pulses needed to observe LIPSS decreases, indicates that this process is more efficient for a shorter time separation between successive pulses. This observation supports the combined action of subsequent pulses as the origin of the positive feedback for LIPSS development. In fact, for a pulse separation longer than $0.1 \mathrm{~s}$ the feedback effect is reduced although is still present at times as long as $1 \mathrm{~s}$. Our results indicate that subsequent cooling after the action of a single pulse may be not complete for $10 \mathrm{~Hz}$ repetition rate, thus successive pulses will lead to an enhanced heating effect. According to eq. 2 this will lead to an increase of roughness by the 
enlarged contribution of the longest wavelength Fourier components which in turn will make possible to reach the longest possible period, close to the irradiating laser wavelength. On the contrary, for lower repetition rates heating caused by a single pulse can be more effectively dissipated before the next one reaches the sample surface and the roughness cannot evolve towards the longest periods. The time intervals between pulses in the range of repetition rates studied are much longer than the thermal diffusion times calculated considering the thermal properties of the pristine polymer and its absorption coefficient. ${ }^{15,}{ }^{22}$ However, it must be considered that the polymer physical properties are modified upon successive irradiation and its optical and thermal properties depend on temperature, thus explaining the difference between the calculated and the experimental observation. This will cause a kind of incubation effect resulting in an increase of the absorption coefficient upon repetitive irradiation, and thus, in an increasing feedback effect and the subsequent reduction of threshold for LIPSS formation. Similar results as the ones reported here, concerning the dependence of structures size and quality on the pulse repetition rate have been previously reported ${ }^{55,56}$ for silicon and calcium fluoride irradiated with femtosecond pulses and repetition rates in the range of $10^{3}-1 \mathrm{~Hz}$. In these works Reif et al. proposed that the way in which the obtained superficial patterns change suggests that at longer separation between pulses the accumulated dose is less than that at short separation, ${ }^{55}$ thus concluding that the feedback effect takes place for separation times of $1 \mathrm{~s}$ and longer. These authors performed calculations on temperature and carrier density and, even if the simulation indicated the existence of a feedback behavior, the time scale was by far shorter than the experimental observation.

The increase of the period obtained for irradiation at $10 \mathrm{~Hz}$ as a function of the number of pulses is also in agreement with an effective feedback effect, i.e. the accumulation of successive 
pulses leads to an increase of the superficial temperature, which in turn generates a softer material with lower superficial viscosity allowing the formation of wider structures characterized by larger periods at larger number of pulses ${ }^{57}$.

\section{CONCLUSIONS}

In situ GISAXS measurements during laser irradiation of spin-coated films of a model polymer, poly(trimethylene terephthalate), have been shown to be feasible by using synchrotron radiation. The analysis of the GISAXS patterns recorded simultaneously to laser irradiation has allowed us to characterize in detail the kinetics of LIPSS formation. The ripples are modelled as forming one dimensional paracrystals with order degree that depends on both the laser fluence and number of pulses. A method based on modelling the periodic structures has been proposed to estimate the paracrystalline order from the in situ data. The role of the laser repetition rate in the development of the superficial nanostructures has been clarified. For high pulse repetition rates $(10 \mathrm{~Hz})$ LIPSS evolve in irradiation time to reach the expected period matching the laser wavelength. For lower pulse repetition rates, 1,2 and $5 \mathrm{~Hz}$, LIPSS formation is less effective and the period of the ripples formed never reaches the value of the laser wavelength. Our results indicate that subsequent cooling of the surface of the polymer film after the action of a single pulse may be not complete for the high repetition rates. In this case the heating effect induced by the next pulse will be enhanced causing an increase of roughness until the longest period is reached. However, for lower repetition rates, the heating induced by a single pulse can be more effectively dissipated and the condition that makes roughness to evolve towards the longest possible period is never reached. This behaviour supports the existence of a feedback effect for LIPSS formation in polymer films. For a pulse separation longer than $0.1 \mathrm{~s}$, the feedback is reduced, although still present at time intervals as long as $1 \mathrm{~s}$. 


\section{AUTHOR INFORMATION}

\section{Corresponding Author}

*E-mail: e.rebollar@iqfr.csic.es

\section{Present Addresses}

+Present address: Department of Physics, University of Surrey, Guildford GU2 7XH, United Kingdom.

\section{ACKNOWLEDGMENT}

The authors gratefully acknowledge financial support from the MINECO (MAT2011-23455, MAT2012-33517 and CTQ 2013-43086-P). E.R., I. M-F. and A.R-R. also thank MINECO for a Ramón y Cajal contract (RYC-2011-08069) and FPI fellowships (BES-2010-030074 and BES2013-062620). We thank Z. Roslaniec and A. Szymczyk for providing the PTT sample and W. Bras for fruitful discussion.

\section{REFERENCES}

(1) Guo, L. J. Nanoimprint lithography: Methods and material requirements. Advanced Materials 2007, 19 (4), 495-513.

(2) Qin, D.; Xia, Y. N.; Whitesides, G. M. Soft lithography for micro- and nanoscale patterning. Nat. Protoc. 2010, 5 (3), 491-502.

(3) Ho, C. C.; Chen, P. Y.; Lin, K. H.; Juan, W. T.; Lee, W. L. Fabrication of Monolayer of Polymer/Nanospheres Hybrid at a Water-Air Interface. ACS Appl. Mater. Interfaces 2011, 3 (2), 204-208.

(4) Schift, H. NaPANIL-Library of Processes, NaPANIL consortium: Berlin, 2012.

(5) Müller-Buschbaum, P.; Bauer, E.; Maurer, E.; Schlögl, K.; Roth, S. V.; Gehrke, R. Route to create large-area ordered polymeric nanochannel arrays. Appl. Phys. Lett. 2006, 88 (8), 083114. 
(6) Müller-Buschbaum, P.; Bauer, E.; Pfister, S.; Roth, S. V.; Burghammer, M.; Riekel, C.; David, C.; Thiele, U. Creation of multi-scale stripe-like patterns in thin polymer blend films. Europhysics Letters 2006, 73 (1), 35-41.

(7) Zhang, P.; Huang, H.-y.; Chen, Y.; Yu, S.; Krywka, C.; Vayalil, S.; Roth, S.; He, T.-b. Preparation of long-range ordered nanostructures in semicrystalline diblock copolymer thin films using micromolding. Chin J Polym Sci 2014, 32 (9), 1188-1198.

(8) Reif, J.; Costache, F.; Henyk, M.; Pandelov, S. V. Ripples revisited: Non-classical morphology at the bottom of femtosecond laser ablation craters in transparent dielectrics. Appl. Surf. Sci. 2002, 197-198 891-895.

(9) Kumar Das, S.; Dufft, D.; Rosenfeld, A.; Bonse, J.; Bock, M.; Grunwald, R. Femtosecondlaser-induced quasiperiodic nanostructures on TiO2 surfaces. J. Appl. Phys. 2009, 105 (8), 084912-5.

(10) Reif, J.; Varlamova, O.; Bounhalli, M.; Muth, M.; Arguirov, T. Nanostructure formation upon femtosecond ablation from silicon: Effect of double pulses. Appl. Surf. Sci. 2012, 258 (23), 9491-9495.

(11) Dufft, D.; Rosenfeld, A.; K. Das, S.; Grunwald, R.; Bonse, J. Femtosecond laser-induced periodic surface structures revisited: A comparative study on ZnO. J. Appl. Phys. 2009, 105 (3), 034908-9.

(12) Bonse, J.; Kruger, J. Pulse number dependence of laser-induced periodic surface structures for femtosecond laser irradiation of silicon. J. Appl. Phys. 2010, 108 (3), 034903-5.

(13) Sanz, M.; Rebollar, E.; Ganeev, R. A.; Castillejo, M. Nanosecond laser-induced periodic surface structures on wide band-gap semiconductors. Appl. Surf. Sci. 2013, 278 (0), 325-329. (14) Rebollar, E.; R. Vazquez de Aldana, J.; Perez-Hernandez, J. A.; Ezquerra, T. A.; Moreno, P.; Castillejo, M. Ultraviolet and infrared femtosecond laser induced periodic surface structures on thin polymer films. Appl. Phys. Lett. 2012, 100 (4), 041106-4.

(15) Rebollar, E.; Pérez, S.; Hernández, J. J.; Martín-Fabiani, I.; Rueda, D. R.; Ezquerra, T. A.; Castillejo, M. Assessment and Formation Mechanism of Laser-Induced Periodic Surface

Structures on Polymer Spin-Coated Films in Real and Reciprocal Space. Langmuir 2011, 27 (9), 5596-5606.

(16) Csete, M.; Marti, O.; Bor, Z. Laser-induced periodic surface structures on different polycarbonate films. Appl. Phys. A 2001, 73 (4), 521-526.

(17) Lazare, S.; Bolle, M.; Cros, A.; Bellard, L. Periodic structuration of polymer surfaces with the excimer laser radiation. Nucl. Inst. Met. Phys. Res.B 1995, 105 (1-4), 159-163.

(18) Rebollar, E.; Vazquez de Aldana, J. R.; Martin-Fabiani, I.; Hernandez, M.; Rueda, D. R.; Ezquerra, T. A.; Domingo, C.; Moreno, P.; Castillejo, M. Assessment of femtosecond laser induced periodic surface structures on polymer films. Phys. Chem. Chem. Phys. 2013, 15 (27), 11287-11298.

(19) Slepicka, P.; Rebollar, E.; Heitz, J.; Svorcik, V. Gold coatings on polyethyleneterephthalate nano-patterned by F-2 laser irradiation. Appl. Surf. Sci. 2008, 254 (11), 3585-3590.

(20) Bäuerle, D. Laser processing and chemistry, Springer-Verlag: Berlin, 2000.

(21) Csete, M.; Bor, Z. Laser-induced periodic surface structure formation on polyethyleneterephthalate. Appl. Surf. Sci. 1998, 133 (1-2), 5-16.

(22) Martín-Fabiani, I.; Rebollar, E.; Pérez, S.; Rueda, D. R.; García-Gutiérrez, M. C.; Szymczyk, A.; Roslaniec, Z.; Castillejo, M.; Ezquerra, T. A. Laser-Induced Periodic Surface Structures Nanofabricated on Poly(trimethylene terephthalate) Spin-Coated Films. Langmuir 2012, 28 (20), 7938-7945. 
(23) Pérez, S.; Rebollar, E.; Oujja, M.; Martín, M.; Castillejo, M. Laser-induced periodic surface structuring of biopolymers. Appl. Phys. A 2013, 110 683-690.

(24) Yadavalli, N. S.; Santer, S. In-situ atomic force microscopy study of the mechanism of surface relief grating formation in photosensitive polymer films. J. Appl. Phys. 2013, 113 (22), 224304.

(25) Yadavalli, N. S.; Saphiannikova, M.; Santer, S. Photosensitive response of azobenzene containing films towards pure intensity or polarization interference patterns. Appl. Phys. Lett.

2014, 105 (5), 051601.

(26) Schwartzkopf, M.; Buffet, A.; Korstgens, V.; Metwalli, E.; Schlage, K.; Benecke, G.; Perlich, J.; Rawolle, M.; Rothkirch, A.; Heidmann, B.; Herzog, G.; Muller-Buschbaum, P.; Rohlsberger, R.; Gehrke, R.; Stribeck, N.; Roth, S. V. From atoms to layers: in situ gold cluster growth kinetics during sputter deposition. Nanoscale 2013, 5 (11), 5053-5062.

(27) Renaud, G.; Lazzari, R.; Leroy, F. Probing surface and interface morphology with Grazing Incidence Small Angle X-Ray Scattering. Surface Science Reports 2009, 64 (8), 255-380.

(28) Narayanan, S.; Lee, D.; Hagman, A.; Li, X.; Wang, J. Particle Dynamics in Polymer-Metal Nanocomposite Thin Films on Nanometer-Length Scales. Phys. Rev. Lett. 2007, 98 (18), 185506.

(29) Muller-Buschbaum, P. The Active Layer Morphology of Organic Solar Cells Probed with Grazing Incidence Scattering Techniques. Adv. Mater. 2014, 26 (46), 7692-7709.

(30) Al-Hussein, M.; Schindler, M.; Ruderer, M. A.; Perlich, J.; Schwartzkopf, M.; Herzog, G.; Heidmann, B.; Buffet, A.; Roth, S. V.; Muller-Buschbaum, P. In Situ X-ray Study of the Structural Evolution of Gold Nano-Domains by Spray Deposition on Thin Conductive P3HT Films. Langmuir 2013, 29 (8), 2490-2497.

(31) Szymezyk, A.; Senderek, E.; Nastalczyk, J.; Roslaniec, Z. New multiblock poly(etherester)s based on poly(trimethylene terephthalate) as rigid segments. Eur. Polym. J. 2008, 44 (2), 436-443.

(32) Martin-Fabiani, I.; Linares, A.; Nogales, A.; Ezquerra, T. A. Dielectric relaxation of poly (trimethylene terephthalate) in a broad range of crystallinity. Polymer 2013, 54 (21), 5892-5898. (33) Bras, W.; Dolbnya, I. P.; Detollenaere, D.; van Tol, R.; Malfois, M.; Greaves, G. N.; Ryan, A. J.; Heeley, E. Recent experiments on a combined small-angle/wide-angle X-ray scattering beam line at the ESRF. J. Appl. Cryst. 2003, 36 791-794.

(34) Roth, S. V.; Dohrmann, R.; Dommach, M.; Kuhlmann, M.; Kroger, I.; Gehrke, R.; Walter, H.; Schroer, C.; Lengeler, B.; Muller-Buschbaum, P. Small-angle options of the upgraded ultrasmall-angle x-ray scattering beamline BW4 at HASYLAB. Rev. Sci. Instrum. 2006, 77 (8), (35) Müller-Buschbaum, P. Applications of Synchrotron Light to Scattering and Diffraction in Materials and Life Sciences. In Lecture Notes in Physics; T. A. Ezquerra; M. C. GarcíaGutiérrez; A. Nogales; M. Gómez; Springer-Verlag: Berlin, 2009; 776, (36) Hammersley, A. FIT2D Website. http://www.esrf.eu/computing/scientific/FIT2D (accessed March 15, 2010).

(37) Hernandez, J. J.; Rueda, D. R.; Garcia-Gutierrez, M. C.; Nogales, A.; Ezquerra, T. A.; Soccio, M.; Lotti, N.; Munari, A. Structure and Morphology of Thin Films of Linear Aliphatic Polyesters Prepared by Spin-Coating. Langmuir 2010, 26 (13), 10731-10737.

(38) Yoneda, Y. Anomalous surface reflection of X rays. Physical Review 1963, 131 (5), 20102013. 
(39) Hernández, J. J.; Rueda, D. R.; García-Gutiérrez, M. C.; Nogales, A.; Ezquerra, T. A.; Soccio, M.; Lotti, N.; Munari, A. Structure and Morphology of Thin Films of Linear Aliphatic Polyesters Prepared by Spin-Coating. Langmuir 2010, 26 (13), 10731-10737.

(40) Kakudo, K.; Kasai, N. X-ray Diffraction by Polymers, Elsevier Publishing Company: Amsterdam, 1972.

(41) Rueda, D. R.; Martin-Fabiani; I.Soccio, M. A.; N.Perez-Murano; F.Rebollar; E.GarciaGutierrez; C.Castillejo, M.; Ezquerra, T. A. Grazing-incidence small-angle X-ray scattering of soft and hard nanofabricated gratings. J. Appl. Cryst. 2012, 45 1038-1045.

(43) Hindeleh, A. M.; Hosemann, R. Microparacrystals-The intermediate stage between crystalline and amorphous. J. Mater. Sci. 1991, 26 (19), 5127-5133.

(44) Ivanov, D. A.; Bar, G.; Dosiere, M.; Koch, M. H. J. A Novel View on Crystallization and Melting of Semirigid Chain Polymers: The Case of Poly (trimethylene terephthalate).

Macromolecules 2008, 41 (23), 9224-9233.

(45) Lazzari, R. IsGISAXS: a program for grazing-incidence small-angle X-ray scattering analysis of supported islands. Journal of Applied Crystallography 2002, 35 406-421.

(46) Stribeck, N. Scattering of Soft Condensed Matter: From Fundaments to Application. In Lecture Notes in Physics; T. A. Ezquerra; M. C. García-Gutiérrez; A. Nogales; M. Gómez; Springer-Verlag: Berlin Heidelberg, 2009; 23-60.

(47) Guosheng, Z.; Fauchet, P. M.; Siegman, A. E. Growth of spontaneous periodic surface structures on solids during laser illumination. Phys. Rev. B 1982, 26 (10), 5366-5381.

(48) Couillard, M.; Borowiec, A.; Haugen, H. K.; Preston, J. S.; Griswold, E. M.; Botton, G. A. Subsurface modifications in indium phosphide induced by single and multiple femtosecond laser pulses: A study on the formation of periodic ripples. Journal of Applied Physics 2007, 101 (3), 033519.

(49) Reif, J.; Costache, F.; Varlamova, O.; Jia, G. B.; Ratzke, M. Self-organized regular surface patterning by pulsed laser ablation. In Physica Status Solidi C - Current Topics in Solid State Physics, Vol 6, No 3; M. Stutzmann; Wiley-V C H Verlag Gmbh: Weinheim, 2009; 6, 681-686. (50) Costache, F.; Eckert, S.; Reif, J. Near-damage threshold femtosecond laser irradiation of dielectric surfaces: desorbed ion kinetics and defect dynamics. Appl. Phys. A-Mater. Sci. Process. 2008, 92 (4), 897-902.

(51) Buff, F. P.; Lovett, R. A.; Stilling.Fh Interfacial density profile for fluids in critical region. Phys. Rev. Lett. 1965, 15 (15), 621-\&.

(52) Müller-Buschbaum, P.; Gutmann, J. S.; Lorenz, C.; Schmitt, T.; Stamm, M. Decay of Interface Correlation in Thin Polymer Films. Macromolecules 1998, 31 (26), 9265-9272.

(53) Mate, C. M.; Toney, M. F.; Leach, K. A. Roughness of thin perfluoropolyether lubricant films: Influence on disk drive technology. IEEE Transactions on Magnetics 2001, 37 (4 I), 1821 1823.

(54) Reiter, G.; de Gennes, P. G. Spin-cast, thin, glassy polymer films: Highly metastable forms of matter. Eur. Phys. J. E 2001, 6 (1), 25-28.

(55) Reif, J.; Varlamova, O.; Bounhalli, M.; Arguirov, T. Long-Time Feedback in the Formation of Self-Organized Nanostructures upon Multipulse Femtosecond Laser Ablation. In International Symposium on High Power Laser Ablation 2010; C. R. Phipps; Amer Inst Physics: Melville, 2010; 1278, 446-456.

(56) Reif, J.; Varlamova, O.; Ratzke, M.; Schade, M.; Leipner, H. S.; Arguirov, T. Multipulse feedback in self-organized ripples formation upon femtosecond laser ablation from silicon. Appl. Phys. A-Mater. Sci. Process. 2010, 101 (2), 361-365. 
(57) Tsutsumi, N.; Fujihara, A. Self-assembled spontaneous structures induced by a pulsed laser on a surface of azobenzene polymer film. Journal of Applied Physics 2007, 101 (3), 033110.

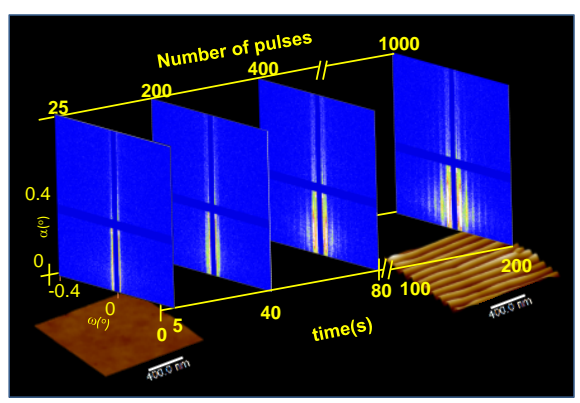

